
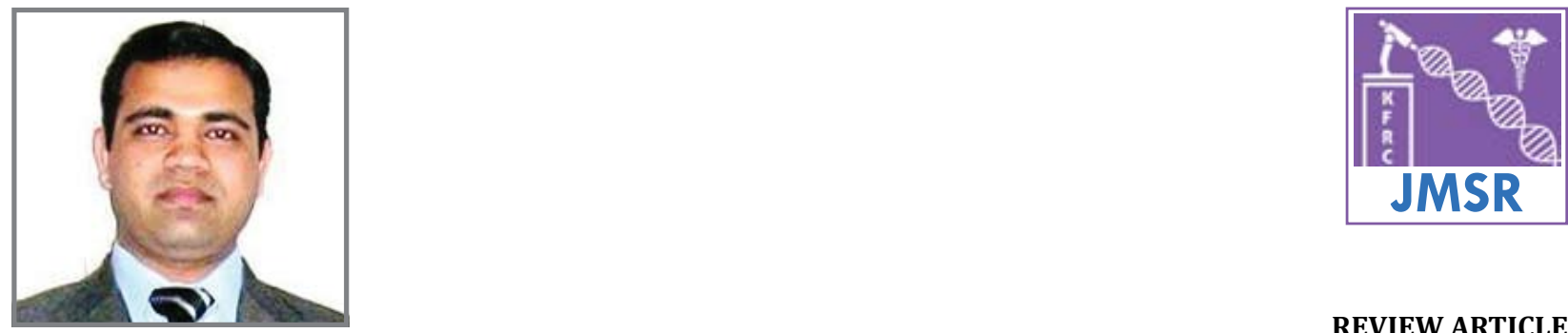

Dr. Vinod Kumar Verma

REVIEW ARTICLE

\title{
Therapeutic potential of different types of stem cells
}

\author{
Dr. Vinod Kumar Verma ${ }^{1,}{ }^{*}$, Dr. Syed Sultan Beevi ${ }^{1}$ and Dr. (Smt.) GVVS. Kanaka Bhushanam ${ }^{1}$ \\ ${ }^{1}$ KIMS Foundation and Research Center, Minister's Road, Secunderabad-500 003, Telangana, India
}

\begin{abstract}
Stem cells are undifferentiated biological cells with the property of self-renewal, can transform into specialized cells and maintain the normal turnover of regenerative organs, such as blood, skin, or intestinal tissues. Stem cells have great possibility in the source of replacement of cells to manage various diseases, conditions, and disabilities such as, stroke, alzheimer's, amyotrophic lateral sclerosis (ALS), spinal cord injury, burns, heart disease, diabetes, arthritis and some genetic disorders. Extensive research is required for better understanding to exploit stem cell is inherent property to cure many debilitating diseases and enhancing overall quality of life.
\end{abstract}

Keywords: Stem cells; Amyotrophic lateral sclerosis; Somatic stem cells

*Corresponding author: Dr. Vinod Kumar Verma, Ph.D., Department of Regenerative Medicine, KIMS Foundation and Research Center, Minister's Road, Secunderabad500 003, Telangana, India. Tel.: 0404488 5058; Email: drvinodkumarverma14@gmail.com

Received 18 June 2014; Revised 21 August 2014; Accepted 01 September 2014

Citation: Vinod Kumar V, Sultan Beevi S, Kanaka Bhushanam GVVS (2014) Therapeutic potential of different types of stem cells. J Med Sci Res 2(4):209-214. DOI: http://dx.doi.org/10.17727/ JMSR.2014/2-037

Copyright: (C) 2014 Vinod Kumar V, et al. This is an open-access article distributed under the terms of the Creative Commons Attribution License, which permits unrestricted use, distribution, and reproduction in any medium, provided the original author and source are credited.

\section{Overview on stem cells Stem cells}

Stem cells are unspecialized cells also known as clonogenic cells and possess the potential to differentiate into one or more specialized cell types [1]. Stemness of stem cells will be maintained in its microenvironment, which is composed of heterologous cell types, extracellular matrix, and soluble factors [2]. Due to its unique differentiation potential they play important role in tissue engineering strategies for the regeneration of diseased, damaged, and missing tissues and even organs [3].

Type of stem cells: In general, stem cells can be divided into four main types. i) Embryonic stem cells: can be isolated from early embryo; ii) Adult stem cells: Present in all tissue/organ types; iii) Induced pluripotent stem cells, or 'reprogrammed' stem cells: have potential similar to embryonic stem cells and made by inserting battery of genes in adult cells; iv) Stem cells derived from cord blood. 


\section{Embryonic stem cells}

Embryonic stem cells (ESCs) are pluripotent stem cells and have great potential to differentiate into specialized cells. These stem cells are derived from the undifferentiated inner cell mass of an early stage of embryo (blastocyst) [4]. These ESCs grow indefinitely in an undifferentiated diploid state when cultured in optimal conditions in the presence of a feeder layer and leukemia inhibitory factor (LIF) [5]. Embryonic stem cells have tremendous potential for therapeutics and research options of variousdiseases. Since 1998 when it was first cultured in lab, several attempts have been made to differentiate ESCs into cardiomyocytes, dopaminergic neurons, pancreatic islets and other tissue types [6]. ESCs have been used in dentistry for oral and craniofacial growth and also generating mucosa, alveolar bone and periodontal tissue etc. [7]. Recently, human ESCs have been cultured under strict quality control conditions, keeping in view of its therapeutic application in human diseases. These 'clinical grade' human ESCs have been approved to carryout clinical trial with the London Project to Cure Blindness. In this trial they have differentiated ESCs into a particular type of eye cell for treatment of patients with age-related macular degeneration. The biotechnology company ACT is also used human ESCs to treat Stargardt's macular dystrophy. Though with much development of ESCs based cell therapy, current challenges include ethical considerations and the need to ensure that ESCs fully differentiate into the required specialized cells before transplantation into patients and should not lead towards teratoma formation. The transplant of ESCs may also pose the problem of graft-vs host reaction after transplantation [8].

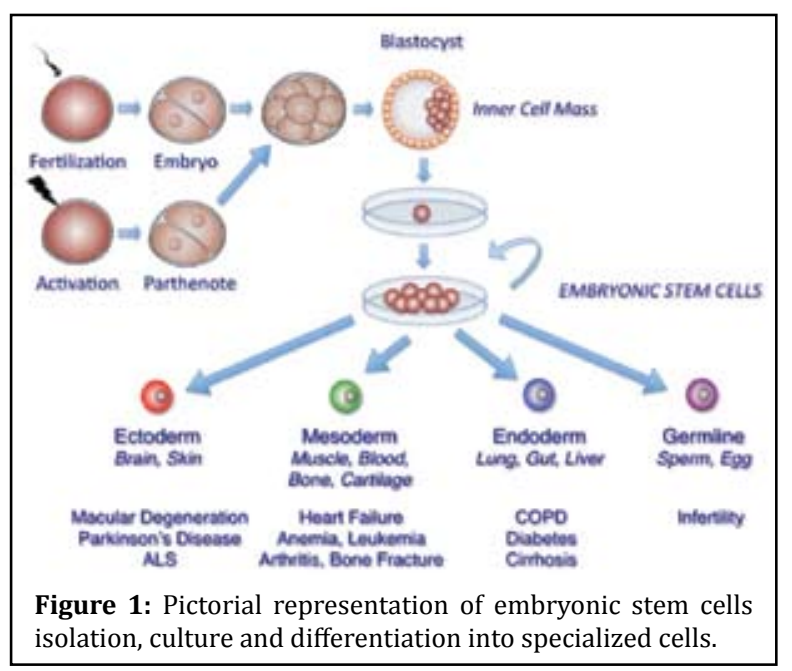

To utilize therapeutic potential of ESCs researchers have to ensure its safety issue utilizing its clinical applications.

\section{Adult/ Somatic stem cells}

Adult stem cells also known as somatic stem cells are undifferentiated cells present in every type of tissue of the body; they divide themselves and regenerate damaged tissues. The adult stem cells have the ability to divide or self-renew indefinitely, and generate all the cell types of the organ from which they originate. Adult stem cells posses the potential of regenerating the entire organ from a few cells. Unlike embryonic stem cells, adult stem cells do not have the problem of graft-vs host reaction after transplantation and not considered to be controversial. The adult stem cells being used for reconstructive and regenerative medicine for various diseases. Few adult stem type, sources and their therapeutic application are explained below.

\section{Hematopoietic stem cells (HSCs)}

Hematopoietic stem cells (HSCs) are derived from mesoderm, have potential to produce all kind of blood cells. They produced and multiply in red bone marrow of most bones. HSCs differentiate into the myeloid (monocytes and macrophages, neutrophils, ba-sophils, eosinophils, erythroc-ytes, megakaryocytes/platelets, and dendritic cells), and lymphoid lineages (T-cells, B-cells and NK-cells) [9]. HSCs are a heterogeneous population contains other cells which have long-term and short-term regeneration capacities. The HSCs population exist three different types of progenitors based on their lymphoid to myeloid progeny (L/M) in blood. Myeloid-biased (My-bi) HSC have low L/M ratio $(>0,<3)$, whereas lymphoid-biased (Ly-bi) show a large ratio $(>10)$. The third category consists of the balanced (Bala) HSC for which $3 \leq \mathrm{L} / \mathrm{M} \leq 10$. Only the myeloid-biased and -balanced HSCs have durable self-renewal properties. More recent advances have resulted in the use of HSCs transplants in the treatment of cancers and other immune system disorders. Several clinical trials and research on animal models has proved that autologous HSCs transplant poses very less risk compared to allogeneic transplant [10]. Since, donor's immune cells sometimes attack the patient's tissues (graftversus-host disease or GVHD) and there is a risk of infection. Hence, during the treatment because the 
patient's own bone marrow cells must be killed with chemotherapy before the transplant can take place.

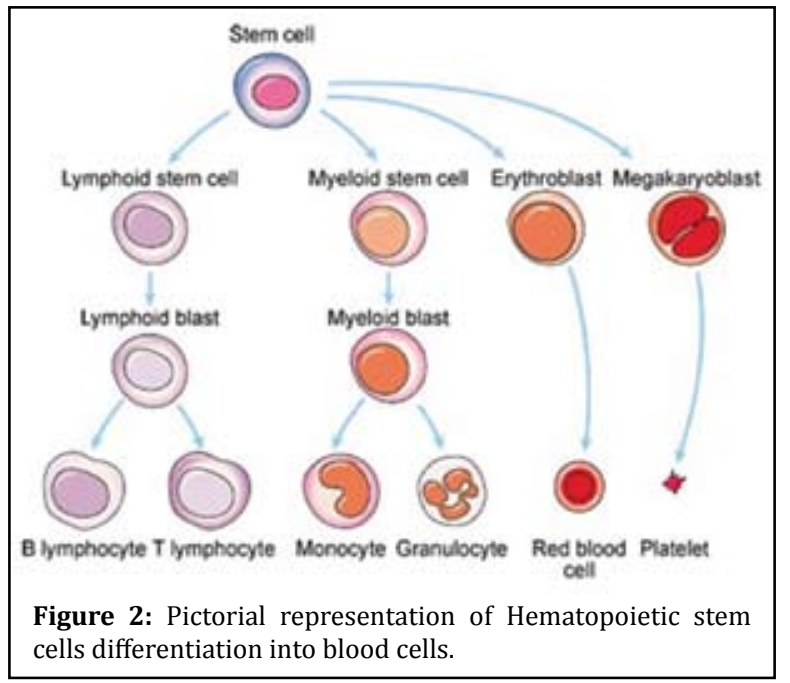

Cord blood-derived multipotent stem cells (CB-SCs) Cord blood-derived multipotent stem cells (CB-SCs) can be harvested from the umbilical cord of a baby after birth. They are identifiable by their ability to attach to the plastic surface of non-tissue culturedtreated Petri dishes. Moreover, CB-SCs possess very low immunogenicity because of diminished expression level of major histocompatibility (MHC) antigens and as a results of that it fails to stimulate the proliferation of allogeneic lymphocytes [11]. The cells can be frozen (cryopreserved) in cell banks and are currently used to treat children with blood disorders such as leukaemia, as well as genetic disorders like Fanconi anaemia. However, cord blood transplant to adults has so far been more challenging and restricted by the number of cells that can be obtained from one umbilical cord and of course immune response may also play a role, still adults have been successfully treated with double cord transplants [12]. The very advantage of cord blood transplants that they have very less chance to be rejected by the immune system because they less immunogenic compared with hematopoietic stem cells. However, for successful transplant cord blood must still be matched to the patient to be successful [13]. Cord blood may also contain stem cell progenitor that can produce other types of specialized cells which can be used for therapy.

\section{Stem cells derived from skin}

Stem cells derived from skin have been used to grow new skin in the lab for severe burn patients.

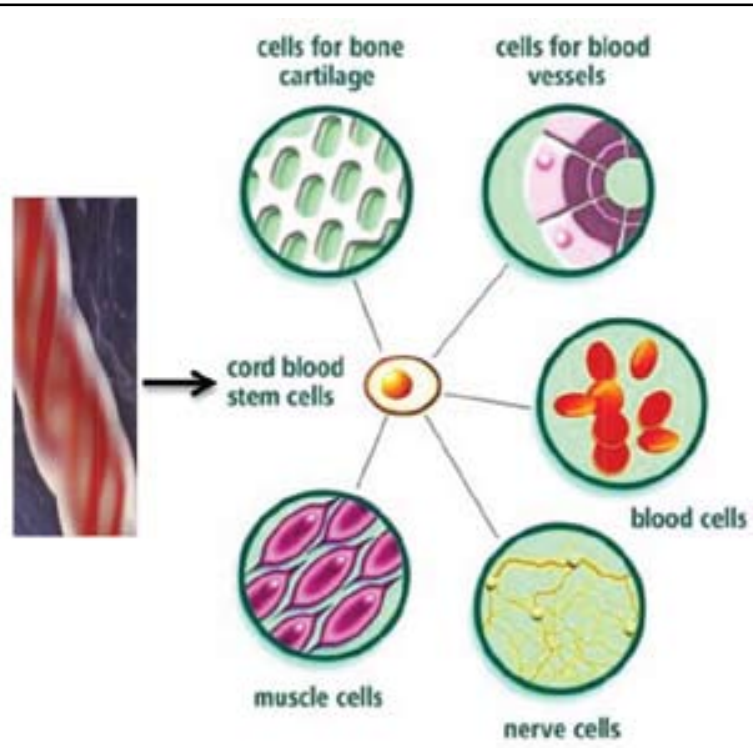

Figure 3: Diagrammatic illustration of cord blood stem cell differentiation into blood cells, nerve cells etc.

The skin sheet grown from stem cells has no hair follicles, sweat glands or sebaceous (oil) glands, so still the technique is far from perfect and further research is needed to improve it [14] Currently, the technique is mainly used to save the lives of patients who have third degree burns over very large areas of their bodies and is only carried out in a few clinical centers.

\section{Limbal stem cells (Stem cells in eye)}

The corneal limbus is the junction of the cornea and the sclera (the white of the eye) which is common site for the occurrence of corneal epithelial neoplasm [15]. Corneoscleral junction possesses maximum population of limbal epithelial stem cells (LESCs). Therapeutic application of LESCs shows in the patient with severely damaged cornea due to chemical burn or other reason, to restoring the sight. However, this can only help patients who have some undamaged limbal stem cells remaining in one of their eyes. The treatment has been shown to be safe and effective in early stage trials. Further studies with larger numbers of patients must now be carried to determine the safety of LESCs in its therapeutic application.

\section{Adipose derived stem cells}

Adipose-derived stem cells provideapromisingfuture in the field of tissue engineering and regenerative medicine. Adipose-derived stem cells (ADSCs) are 


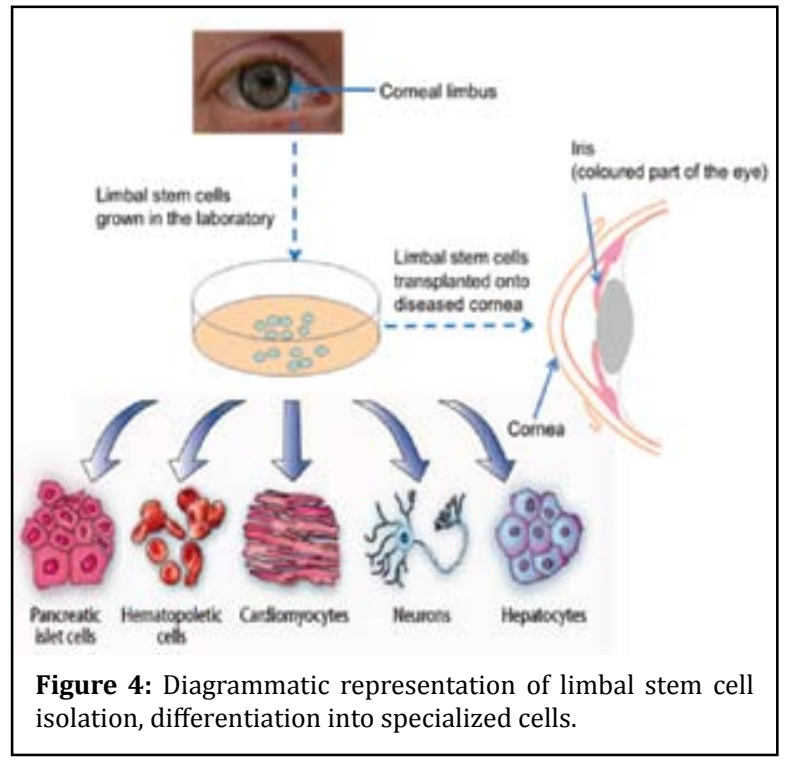

a potential cell source for stem cell-based therapy because they have enormous trilineage (adipocytes, osteoblasts, chondrocytes) differentiation capacity into specialized cell types [16]. ADSCs can be found in white adipose tissue, including subcutaneous and omental fat. ADSC can be isolated from liposuction by enzymatic digestion or mechanical and expanded in in vitro until they enter into the differentiation process to specific cell lineages. Thus, ADSCs would be a valuable stem cell source for clinical use, with fewer restrictions compared to other cell sources. Adipose stem cells have been utilized in studies addressing osteoarthritis, diabetes mellitus, heart disease, and soft tissue regeneration and reconstruction after mastectomy and facial repair [17].

\section{Mesenchymal stem cells (MSCs)}

Mesenchymal stem cells are multi potent stromal cells possess trilineage differentiation potential i.e. osteoblasts, chondrocytes, and adipocytes. MSCs are derived from several types of tissues like bone marrow, placenta, umbilical cord blood, adipose tissue, adult muscle and dental pulp of deciduous baby teeth. The umbilical cord which is normally thrown away after the birth of the newborn is proven very good source of MSCs [18]. These MSCs have more primitive properties than other adult MSCs that make them very useful source for clinical applications. The MSCs eventually form minimum of 29 different unique end organs like enamel, dentin, blood vessels, dental pulp, and nervous tissue. Several laboratory investigations shown that adipose tissue is one of the richest sources of MSCs and

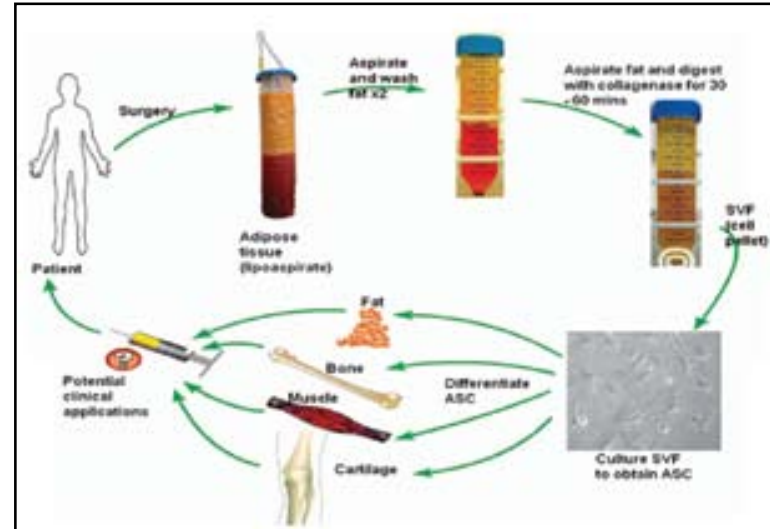

Figure 5: Demonstration of isolation of adipose derived stem cell isolation by enzymatic disintegration methods, and differentiation into specialized cells.

contains 500 times more stem cells in 1 gram of fat than in 1 gram of aspirated bone marrow. As adipose tissue is thrown away as a waste after surgery, active researches are going on to utilize this for stem cell isolation and its application in therapy.

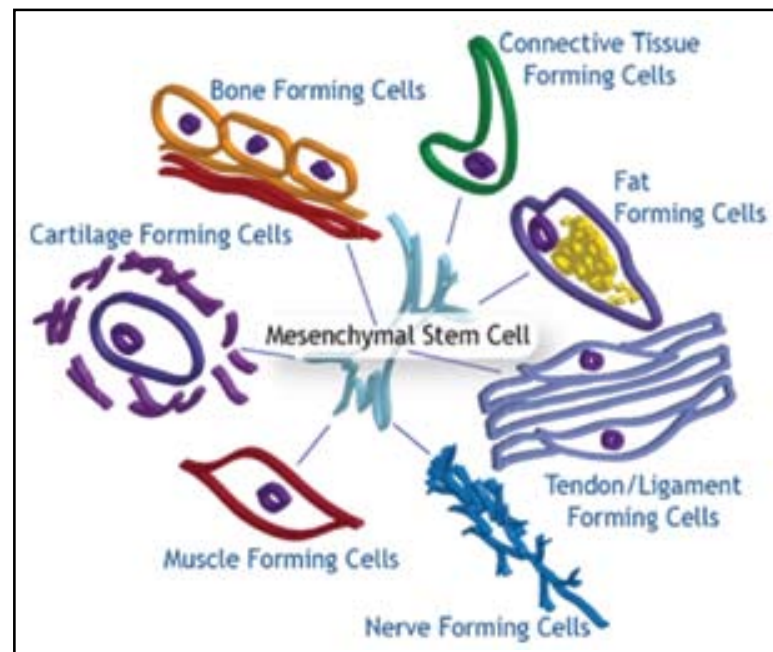

Figure 6: Pictorial sketches of mesenchymal stem cell their potential differentiation into specialized cells.

MSCs can be characterized by presence of CD73, CD90 and CD105, while absence of CD11b, CD14, CD19, CD34, CD45, CD79a and HLA-DR surface markers [19]. Several claims have been made that MSCs can avoid detection by the immune system because avoid allorecognition, interference with dendritic cell and T-cell function, and generate a local immunosuppressive microenvironment by secreting selective cytokines [20]. Due to this property of MSCs can be transplanted from one person into another with little or no risk of rejection. It has also been shown that the immunomodulatory function of human MSC is enhanced when the cells are exposed 
to an inflammatory environment characterised by the presence of elevated local interferon-gamma levels. It has also been suggested that MSCs may be able to affect immune responses in the body to reduce inflammation and help treat transplant rejection or autoimmune diseases. Some clinical trials are investigating the safety and effectiveness of MSC treatments for repairing bone or cartilage [21]. Other trials are investigating whether MSCs might help repair blood vessel damage linked to heart attacks or diseases such as critical limb ischaemia, but still further research is required to establish therapeutic efficacy [22].

\section{Induced pluripotent stem cells (iPS cells)}

Induced pluripotent stem cells are pluripotent stem cell that can be generated directly adult cells by 'reprogramming' into cells that behave like embryonic stem cells [23]. The iPS cells were first generated by Shinya Yamanakain Kyoto, Japan, in 2006. In this technology he introduced four specific transcription factors which could convert adult cells into pluripotent stem cells for that he was awarded Nobel Prize along with Sir John Gurdon in 2012 [24]. Now the iPS has extensive implications in disease research therapy. This finding also allows us to understand the mechanism of disease occurrence and designing the therapeutics. Researchers have generated brain cells from iPSCs made from skin samples belonging to patients with neurological disorders such as Down's syndrome or Parkinson's disease [25]. These stem cells can be used for wide range of disease research and their therapy. However, this technology is very new and use of iPSCs in cell therapy is largely theoretical. Since, these techniques involve genetic modification based on viral transfection, which can sometimes result in the cells forming tumours and may lead towards some viral based infections [26].

\section{Stem cell processing}

The processing of stem cells are important in evaluating a therapeutic efficacy of stem cells in the patient. Basically stem cells are processed by three ways. 1 . Stem cells are processed from bone marrow aspirate (autologous) by density gradient centrifugation where heterogeneous population of monocular cells separated in a layer called buffy coat. These cells are isolated a few hundred and reinfused back into the patient. 2 . Here stem are

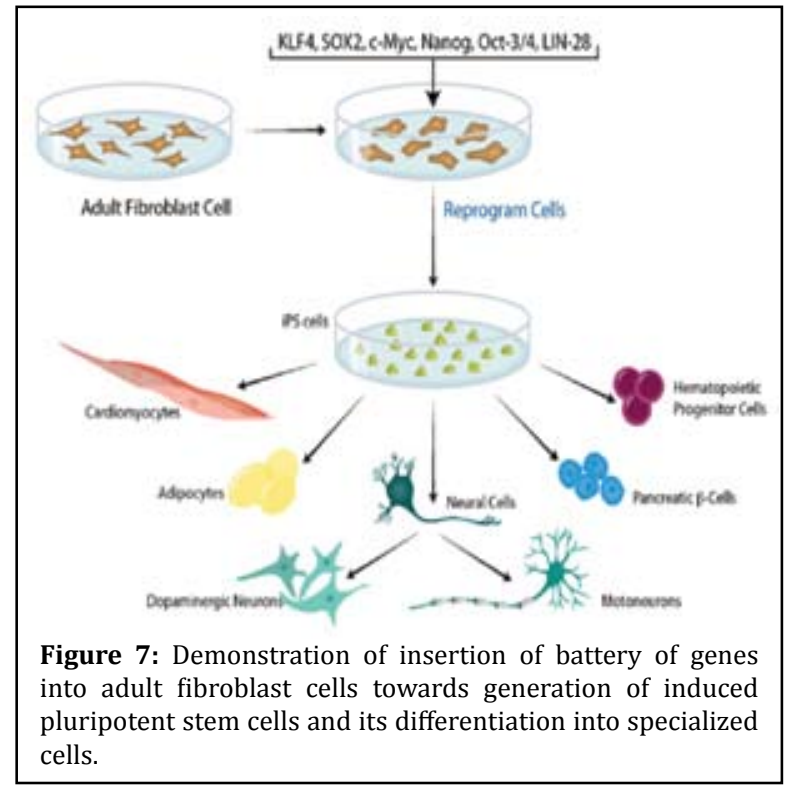

processed by pre-conditioning i.e. stem isolation after administration of growth factors for duration of a few hours to a few days which allows the stem cells to expand and multiply. Pre-conditioning is required collect more number of stem cells for injections. In this method stem cells are cultured and multiplied to several million in laboratory over the course of a few weeks.

\section{Delivery of stem cells}

While IV delivery is attractive because of the low level of expertise and expense it takes to deliver cells, studies have consistently shown that adult stem cells delivered in this fashion are trapped in the lungs (pulmonary first pass effect). Of even more concern is a recent study showing that for patients considering the use of stem cells to treat CNS disorders, only about 1 in 200,000 cells injected via an IV route reaches the CNS (1.5-3.7\% made it past the lungs, $0.295 \%$ made it to the carotid artery, and $0.0005 \%$ made it past the blood brain barrier into the CNS). At this point, until these pulmonary first pass issues are worked out, credible stem cell delivery is local. This means placing cells directly into the tissue or into the arterial circulation that directly supplies the tissue. In addition, for orthopedic applications (and likely for others), it's hyper-local, meaning that placement of cells into one part of the joint may provide results; whereas non-specific placement in the joint provides no results. The same holds true for cardiac placement. Placement in the cardiac arterial circulation will get some cells to the site, 
placement generally in the muscle will get more cells to the site, and placement in the transitional zone between the oxygenated muscle and the necrotic zone (transitional zone) will guarantee better cell survival and more cells that are capable of repair.

\section{Conclusion}

Stem cells are important tools for disease research and offer great potential for use in the clinic. Although embryonic stem cell has limitations yet they are currently being used for clinical trials in animals as well as in human's therapy. Adult stem cells are known for less immunogenic they hold lot of therapeutic potential for several diseases. Meanwhile, induced pluripotent stem cells are already of great use in research, but a lot of work is needed before they can be considered for use in the clinic.

\section{Conflict of interest}

The authors declare no conflict of interest.

\section{References}

1. Chopra H, Kumar M, Shetty S. Stem cells-the hidden treasure. Dent Res J (Isfahan): 2013; 10(4): 421-427.

2. Rachel R Stine, Erika L Matunis. Stem Cell Competition: Finding Balance in the Niche. Trends Cell Biol. 2013; 23(8):357-364.

3. Gadjanski I, Spiller K, Vunjak-Novakovic G. Time-dependent processes in stem cell-based tissue engineering of articular cartilage. Stem Cell Rev. 2012; 8(3): 863-881.

4. Kingham E, Richard OC. Embryonic and Induced Pluripotent Stem Cells: Understanding, Creating, and Exploiting the Nano-Niche for Regenerative Medicine. Oreffo ACS Nano. 2013; 7(3): 1867-1881.

5. Hirai H, Karian P, Kikyo N. Regulation of embryonic stem cell self-renewal and pluripotency by leukaemia inhibitory factor. Biochem J. 2011; 438(1):11-23.

6. Steve KW Oh, Andre BH Choo. human embryonic stem cell technology: Large scale cell amplification and differentiation. Cytotechnology 2006; 50(1-3):181-190.

7. Pagni G, Kaigler D, Rasperini G, Ortiz G, Bartel R, et al. Bone repair cells for craniofacial regeneration. Adv Drug Deliv Rev. 2012; 64(12):1310-1319.

8. Kim EM, Stultz R, Bonde S, Zavazava N. Embryonic stem cell-derived $\mathrm{T}$ cells induce lethal graft-versus-host disease and reject allogenic skin grafts upon thymic selection. Am J Transplant. 2012; 12(3):600-609.

9. Kondo M. Lymphoid and myeloid lineage commitment in multipotent hematopoietic progenitorsImmunol Rev. 2010; 238(1):37-46.

10. Atkins HL, Muraro PA, Van Laar JM, Pavletic SZ. Autologous hematopoietic stem cell transplantation for autoimmune disease-is it now ready for prime time? Biol Blood Marrow Transplant. 2012; 18(1 Suppl):S177-S183.
11. Munoz J, Shah N, Rezvani K, Hosing C, Bollard CM, et al. Concise Review: Umbilical Cord Blood Transplantation: Past, Present, and Future. Stem Cells Transl Med. 2014; 2014-2015.

12. Wagner JE Jr, Eapen M, Carter S, Wang Y, Schultz KR, et al. One-unit versus two-unit cord-blood transplantation for hematologic cancers. Blood and Marrow Transplant Clinical Trials Network. N Engl J Med. 2014; 371(18):1685-1694.

13. Wang L, Xiao Q, Wang CH, Li X, Luo SQ et al. Vasoactive intestinal polypeptide suppresses proliferation of human cord blood-derived hematopoietic progenitorcells by increasing TNF- $\alpha$ and TGF- $\beta$ production in the liver. Genet Mol Res. 2014; 13(4):9032-9043.

14. Perdoni C, McGrath J.A, Tolar J. Preconditioning of mesenchymal stem cells for improved transplantation efficacy in recessive dystrophic epidermolysis bullosa. Stem Cell Res Ther. 2014; 5(6):121.

15. Huang $M$, Wang $B$, Wan $P$, Liang $X$, Wang $X$, et al. Roles of limbal microvascular net and limbal stroma in regulating maintenance of limbal epithelial stem cells. Cell Tissue Res:2014.

16. Dossena M, Bedini G, Rusmini P, Giorgetti E, Canazza A, et al. Human adipose-derived mesenchymal stem cells as a new model of spinal and bulbar muscular atrophy. PLoS One 2014; 9(11).

17. Cruz AC, Caon T, Menin A, Granato R, Boabaid F, et al. Adipose-derived stem cells incorporated into platelet-rich plasma improved bone regeneration and maturation in vivo. Dent Traumatol. 2014;

18. Harkin DG, Foyn L, Bray LJ, Sutherland AJ, Li FJ, et al. Can Mesenchymal Stromal Cells Differentiate into Corneal Cells? A Systematic Review of Published Data. Stem Cells. 2014;

19. Bogdanova A, Berzins U, Nikulshin S, Skrastina D, Ezerta A, et al. Characterization of human adipose derived stem cells cultured in autologous serum after subsequent passaging and long term cryopreservation. J Stem Cells. 2014; 9(3):135-148.

20. Sadeghi L, Kamali-Sarvestani E, Azarpira N, Shariati M, Karimi MH. Immunomodulatory effects of mice mesenchymal stem cells on maturation and activation of dendritic cells. Iran J Immunol. 2014; 11(3):177-188.

21. Doeppner TR, Hermann DM. Stem cell-based treatments against stroke: observations from human proof-of-concept studies and considerations regarding clinical applicability. Front Cell Neurosci. 2014; 8:357.

22. Du S, Guan J, Mao G, Liu Y, Ma S, et al. Intra-arterial delivery of human bone marrow mesenchymal stem cells is a safe and effective way to treat cerebral ischemia in rats. Cell Transplant. 2014.

23. Byrne SM, Mali P, Church GM. Genome editing in human stem cells. Methods Enzymol. 2014; 546:119-138.

24. Nakagawa M, Yamanaka S. Reprogramming of somatic cells to pluripotency. 2010; 695:215-124

25. Johnson-Kerner BL, Ahmad FS, Diaz AG, Greene JP, Gray $S J$, et al. Intermediate filament protein accumulation in motor neurons derived from giant axonal neuropathy iPSCs rescued by restoration of gigaxonin. Hum Mol Genet: 2014 .

26. Hu K. All roads lead to induced pluripotent stem cells: the technologies of iPSC generation. Stem Cells Dev: 2014; 23(12):1285-1300. 